\title{
Understanding intermediate-band solar cells
}

\author{
Antonio Luque ,Antonio Marti and Colin Stanley
}

\begin{abstract}
The intermediate-band solar cell is designed to provide a large photogenerated current while maintaining a high output voltage. To make this possible, these cells incorporate an energy band that is partially filled with electrons within the forbidden bandgap of a semiconductor. Photons with insufficient energy to pump electrons from the valence band to the conduction band can use this intermediate band as a stepping stone to generate an electron-hole pair. Nanostructured materials and certain alloys have been employed in the practical implementation of intermediate-band solar cells, although challenges still remain for realizing practical devices. Here we offer our present understanding of intermediate-band solar cells, as well as a review of the different approaches pursed for their practical implementation. We also discuss how best to resolve the remaining technical issues.
\end{abstract}

$\mathrm{T}$ he intermediate-band (IB) solar cell consists of an IB material sandwiched between two ordinary $\mathrm{n}$ - and p-type semiconductors ${ }^{1}$, which act as selective contacts to the conduction band (CB) and valence band (VB), respectively (Fig. 1a). In an IB material, sub-bandgap energy photons are absorbed through transitions from the VB to the IB and from the IB to the $\mathrm{CB}$, which together add up to the current of conventional photons absorbed through the VB-CB transition. In $1997^{2}$, researchers used hypotheses similar to those adopted by Shockley and Queisser in $1961^{3}$ to derive a detailed balance-limiting efficiency of $63 \%$ for the IB solar cell, at isotropic sunlight illumination (concentration of 46,050 suns) and assuming Sun and Earth temperatures to be $6,000 \mathrm{~K}$ and $300 \mathrm{~K}$, respectively (Fig. 1b). This work also presented the Shockley-Queisser limiting efficiency of $41 \%$ for single-gap solar cells operating under the same conditions $s^{2}$. The limiting efficiency of the IB solar cell is similar to that of a triple-junction solar cell connected in series. However, the IB can be regarded ${ }^{4}$ as a set of two cells connected in series (corresponding to the VB-IB and IB$\mathrm{CB}$ transitions) and one in parallel (corresponding to the $\mathrm{VB}-\mathrm{CB}$ transition). This provides the IB solar cell with additional tolerance to changes in the solar spectrum.

An optimal IB solar cell has a total bandgap of about $1.95 \mathrm{eV}$, which is split by the IB into two sub-bandgaps of approximately $0.71 \mathrm{eV}$ and $1.24 \mathrm{eV}$. The quasi-Fermi levels (QFLs) or electrochemical potentials of the electrons in the different bands are usually close to the edges of the bands. Because the voltage of any solar cell is the difference between the $\mathrm{CB}$ QFL at the electrode in contact with the n-type side and the VB QFL at the electrode in contact with the p-type side, the maximum photovoltage of the IB solar cell is limited to $1.95 \mathrm{eV}$, although it is still capable of absorbing photons of energy above $0.71 \mathrm{eV}$. In contrast, single-gap solar cells cannot supply a voltage greater than the lowest photon energy they can absorb. IB solar cells can deliver a high photovoltage by absorbing two subbandgap photons to produce one high energy electron; the laws of thermodynamics would be violated if this were not the case.

The concept of putting levels in the middle of a bandgap to increase the maximum photocurrent was proposed by solar cell researchers in the mid-twentieth century $y^{5}$. However, the idea was initially rejected because it was believed that these centres would cause non-radiative recombination, which could counteract the benefits of the current gain. Later we will briefly review the basic concepts used to inhibit the non-radiative recombination introduced by these centres. In addition, researchers originally did not realize the importance of splitting the Fermi level into three separate QFLs to preserve the high output voltage of the cell. Subsequent refinements to the initial detailed balance analysis of the IB solar cell ${ }^{4,6-8}$ have confirmed its potential for high efficiency. It is the high value of the IB solar cell's limiting efficiency that has attracted many scientists to work in this field.

\section{Quantum dot IB solar cells}

The energy levels of the confined states in a quantum dot (QD) can be used as an $\mathrm{IB}^{9}$. The first solar cells based on this concept were developed in $2004^{10}$. Figure 1c shows a cell of this type, where molecular beam epitaxy has been used to grow InAs QDs in a matrix of GaAs in the Stranski-Krastanov mode. Figure 2a labels the confined states in a QD (described later), which have been calculated by assuming the QD to be a three-dimensional box. In this plot, the IB corresponds to the CB level labelled $(1,1,1)$. Silicon doping of the barrier material provides the electrons necessary to partially fill the $\mathrm{IB}$, thus making available both empty states to receive electrons from the VB and full states to supply them to the $\mathrm{CB}$.

The quantum efficiency plot in Fig. $1 \mathrm{~d}^{11}$ shows a sub-bandgap current in the IB solar cell that is absent in the reference cell. The small value of this current corresponds to the small number of QD layers introduced - only ten. Unfortunately, increasing the number of QD layers causes an accumulation of strain that seriously damages the cell's structure. It is strain arising from the lattice mismatch between GaAs $(0.567 \mathrm{~nm})$ and $\operatorname{InAs}(0.604 \mathrm{~nm})$ that promotes the Stranski-Krastanov mode of growth. Hubbard and co-workers found that the inclusion of $\mathrm{GaP}$ layers compensated for this strain, allowing them to increase the sub-bandgap current using only five QD layers ${ }^{12}$. Okada and co-workers added layers of GaNAs to achieve strain compensation ${ }^{13}$, but this material has a smaller bandgap than GaAs. Thus, although the cell current increased, its voltage was limited to the GaNAs bandgap. Other groups have also made IB solar cells based on QDs ${ }^{14-20}$; Blokhin et al. ${ }^{17}$ obtained efficiencies of over $18 \%$, and Bailey et al. ${ }^{18}$ achieved open-circuit voltages close to $1 \mathrm{~V}$. However, all of these cells exhibited a reduction in open-circuit voltage with respect to reference cells of the same technology without QDs, as shown in Fig. 1e, leading to a reduction in efficiency against the intended result. This issue will be considered later in this Progress Article, although it is worth noting that the bandgaps of InAs/GaAs cells are far from the optimum values for the implementation of an ideal IB solar cell. InAs/GaAs QDs remain valuable experimental tools, however, as their technology is well established.

Sub-bandgap photocurrent. The small sub-bandgap photocurrent in a QD IB solar cell results primarily from the low density of QDs. However, QDs are quite large nanostructures compared with atoms, 
a

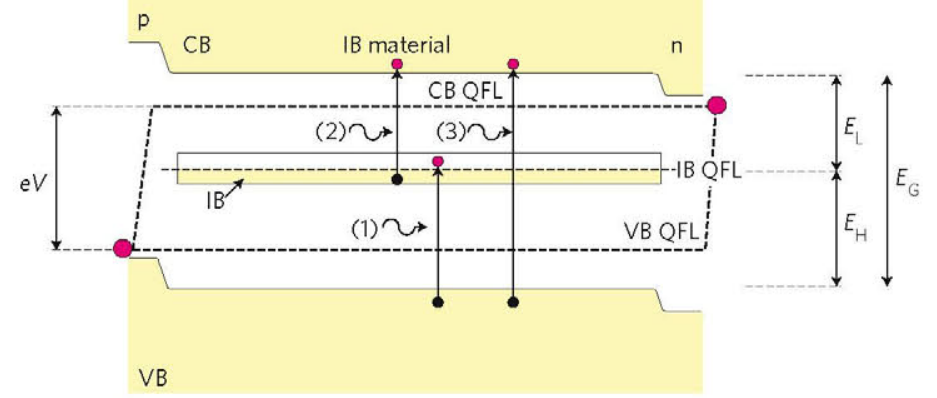

b

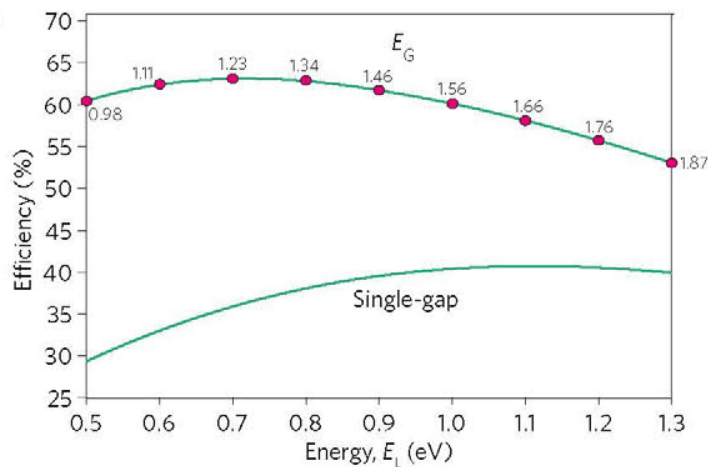

c
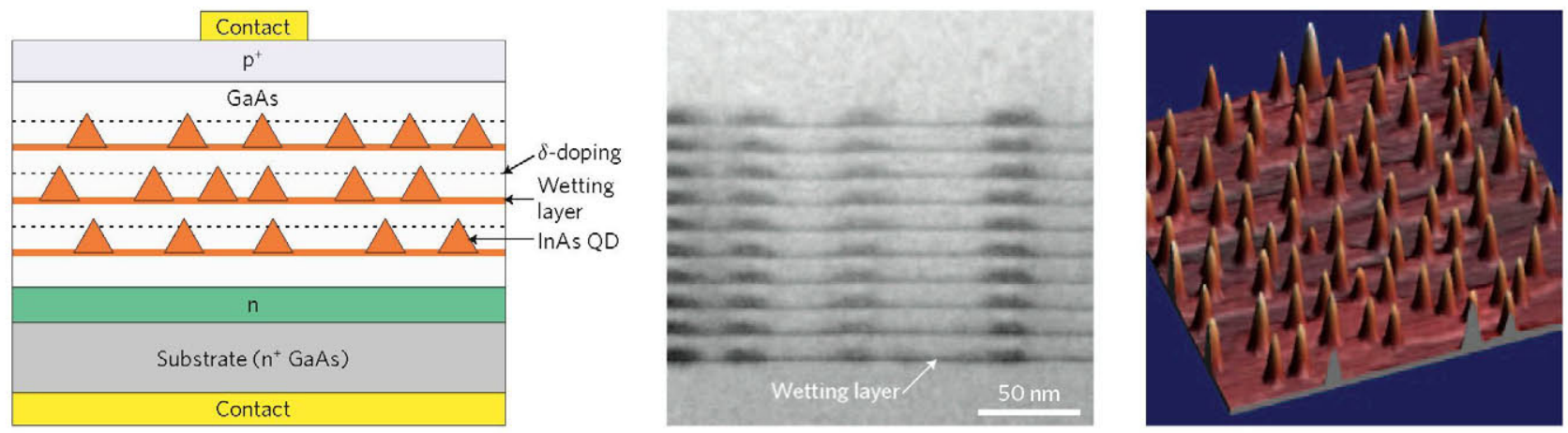

d

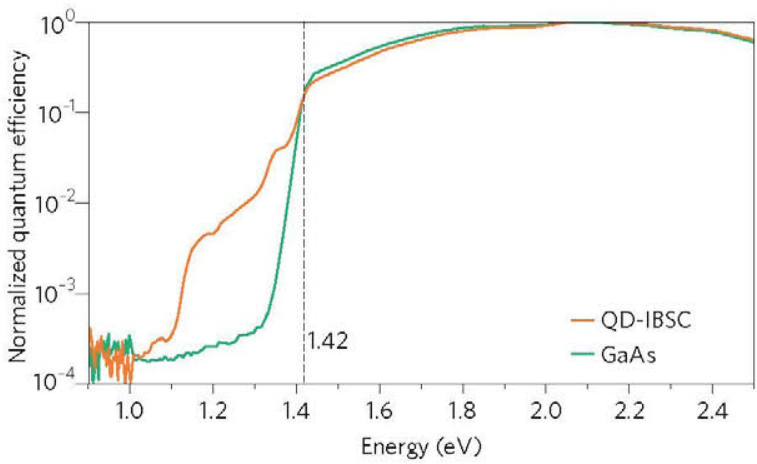

e

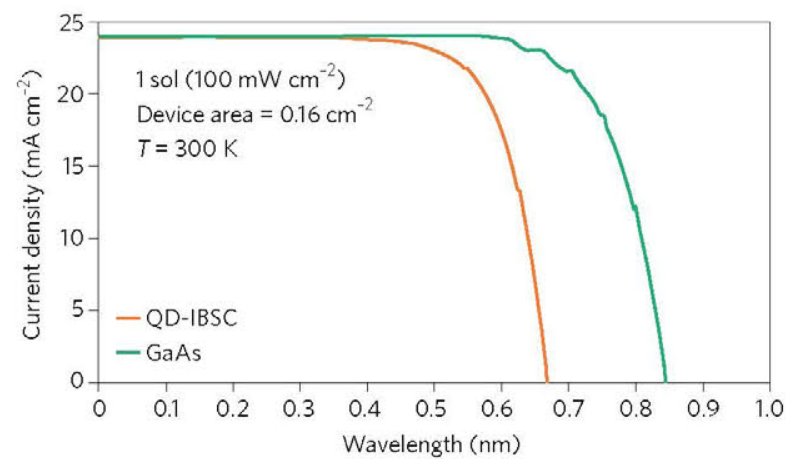

Figure 1 | Theory of the IB solar cell and basic results. $\mathbf{a}$, Band diagram of an IB solar cell, showing the bandgap $E_{G}$, the sub-bandgaps $E_{H}$ and $E_{L}$, as well as the CB, IB and VB QFLs. (1) and (2) represent below-bandgap photon absorption; (3) represents above-bandgap photon absorption. b, Limiting efficiency for IB and single-gap cells as a function of $E_{1}$. $c$, Schematic of a QD IB solar cell, together with a transmission electron microscope image of the QDs and an atomic force microscopy image of a layer of QDs. d, Quantum efficiency of an QD-based IB solar cell (QD-IBSC) and a control (GaAs) solar cell. The response for the QD-IBSC is below the GaAs bandgap (1.42 eV). e, Current-voltage curves of a QD-IBSC and a control (GaAs) solar cell. Figure reproduced with permission from: b, ref. 2, (c) 1997 APS; c, left,right, ref. 10, (c) 2004 AIP; c, middle, ref. 53, (c) 2007 AIP; d, ref. 11, (c) 2008 Elsevier; e, ref. 10, (c) 2004 AIP.

and we might expect that each QD absorbs light rather effectively. Modelling becomes necessary to gain a deeper insight of this issue. Substantially higher QD densities can be achieved by growing QDs on (311) substrates ${ }^{21}$ instead of on (100) substrates, but this refinement has not yet been applied to cell manufacturing.

In a semiconductor QD structure, scientists usually assume that the IB corresponds to the CB state with the lowest energy confined around the QD (labelled $(1,1,1)$ in Fig. 2 a), as opposed to states that extend across the entire semiconductor. InAs QDs in GaAs have a shape that is often approximated as a short square-base truncated pyramid. The impact of this shape on the performance of the QD IB solar cell, although often considered to be of secondary importance, is an issue that can complicate the formulation of a simple model from which the main aspects related to photon absorption in QDs can be understood. In this regard, we will consider a QD represented by a short parallelepiped with a square base. In the $\mathrm{k} \bullet$ p quantum model ${ }^{22}$, the $\mathrm{CB}$ minimum of InAs is lower than that of GaAs and induces a potential well that extends throughout the whole parallelepiped. This potential profile is shown in Fig. $2 \mathrm{a}$ as a grey line. To calculate the CB states, researchers can use the one-band $\mathrm{k} \cdot \mathrm{p}$ approximation, which involves substituting the electron mass by the $\mathrm{CB}$ effective mass and setting the potential to be equal to the potential barrier caused by the QD ${ }^{23}$. This allows the QD energy states and wavefunctions to be calculated, together with the optical transitions from the partially filled IB $(\mathrm{CB}(1,1,1)$ state $)$ to the other localized and extended states in the $\mathrm{CB}$. Confined energy $\mathrm{CB}$ states can be seen in Fig. 2a as $\mathrm{CB}$ levels, and the absorption coefficients are shown in Fig. $2 \mathrm{~d}$ for several illumination conditions ${ }^{24}$.

The study of the VB is much more intricate because it contains light-hole (LH), heavy-hole (HH) and split-off (SO) bands ${ }^{22}$, each having negative effective masses and pedestals instead of well potentials (associated with the VB top). Pedestals are required to 
a
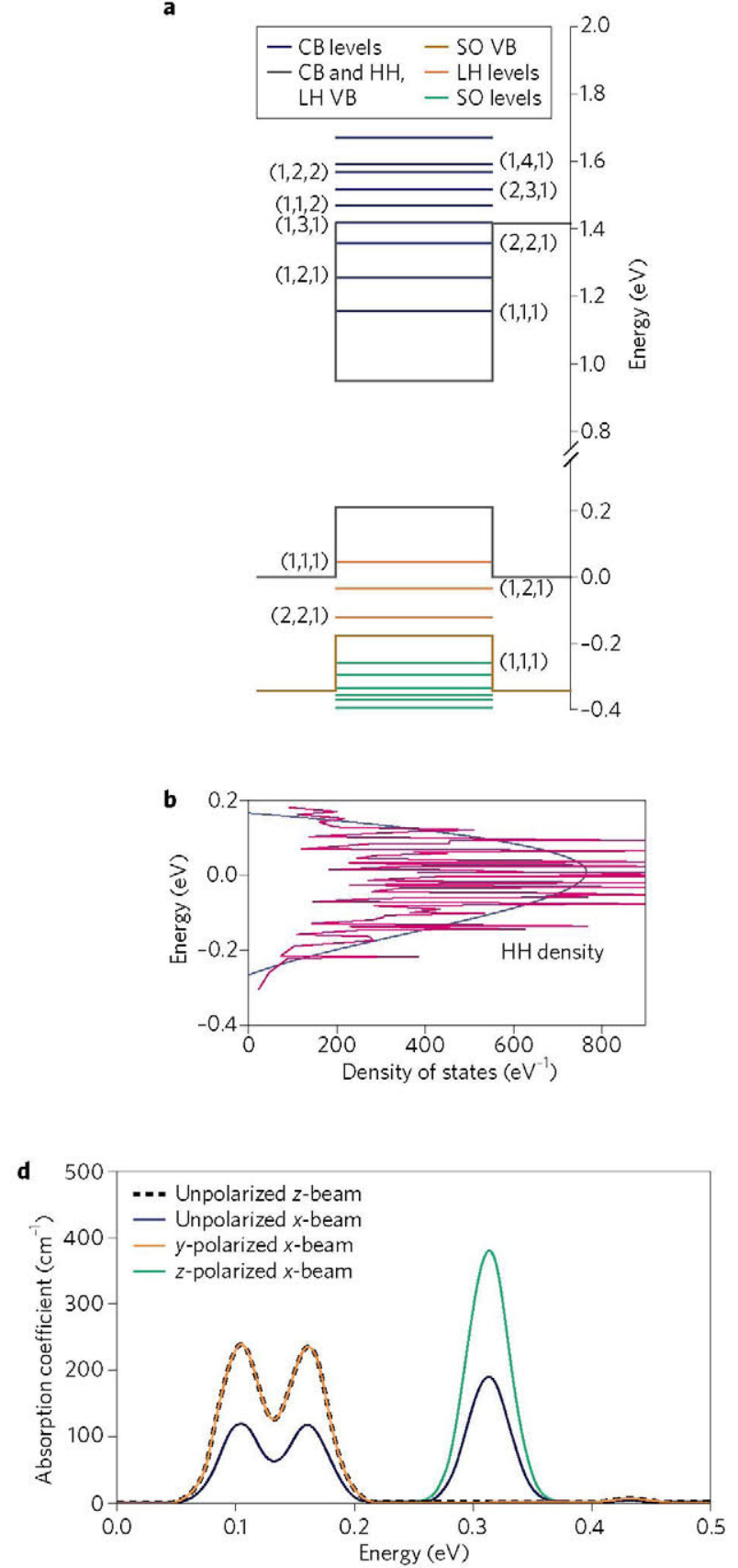

c

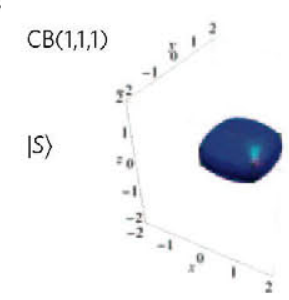

$|X\rangle$

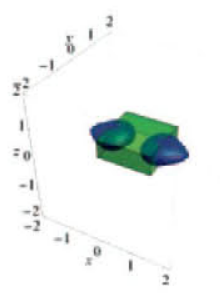

$|Y\rangle$

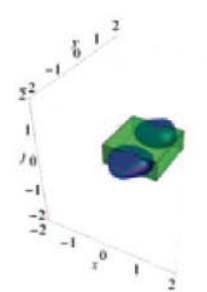

|Z)

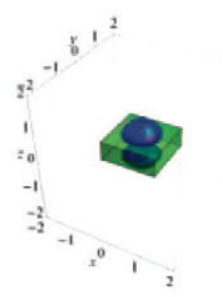

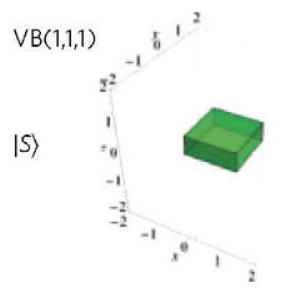

$|X\rangle$

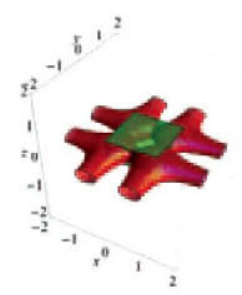

|Y

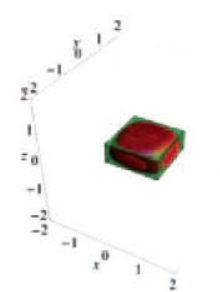

$|Z\rangle$

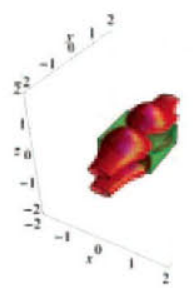

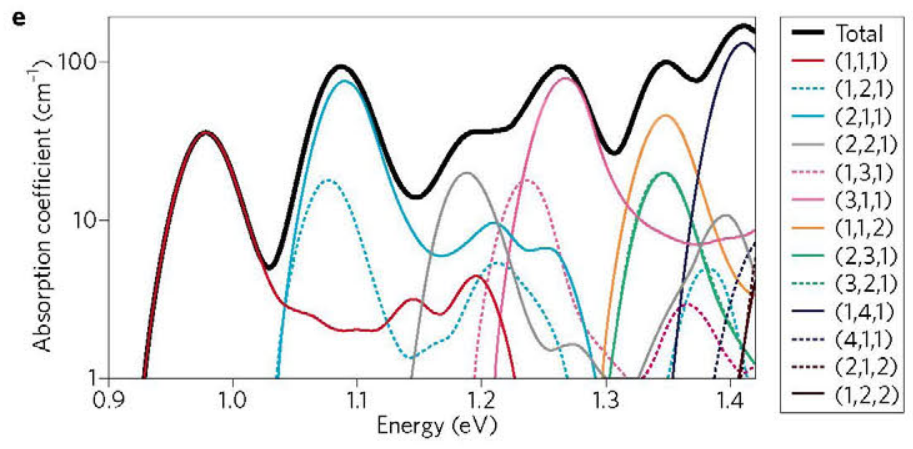

Figure 2 | Energy levels and wavefunctions of a QD whose shape is assumed to be a three-dimensional parallelepiped. Knowledge of these wavefunctions allows the relevant absorption coefficients to be calculated. $\mathbf{a}$, Energy levels for the $\mathrm{CB}$ and the different VBs. The IB corresponds to the state labelled $\mathrm{CB}(1,1,1)$. b. Energy density of states for the $\mathrm{HH}$ states. $\mathbf{c}$, Projections on the $|S\rangle,|X\rangle,|Y\rangle$ and $|Z\rangle$ involutes for the $\mathrm{CB}(1,1,1)($ left $)$ and $\mathrm{HH}(1,1,1)$ (right) states. d, Absorption coefficient for the IB-CB transitions to bounded states, plotted from data in ref. 24 . The results correspond to the case in which the $C B(1,1,1)$ state is half-filled with electrons. e, Absorption coefficient for the bounded VB-IB transitions of a non-polarized beam in the $z$ direction. The quantum numbers refer to the final state. This assumes that the $V B$ states are full of electrons, the $C B$ states are empty and the IB state $C B(1,1,1)$ is half-filled. Figure reproduced with permission from ref. 25, (C) 2011 Elsevier.

confine negative mass quasi-particles, which, in practice, can be treated like positive effective mass particles in wells. This is equivalent to inverting the direction of the energy axis for the VB states, at which point the description becomes the same as for $\mathrm{CB}$ states. Energy levels for the LH and SO bands are therefore also represented in Fig. $2 \mathrm{a}^{25}$. The large effective mass of the HH band means it contains many states. These states are better described by their density, as shown in Fig. 2b, rather than by the levels themselves.
A simplified treatment of the $\mathrm{VB}$ requires a four-band $\mathrm{k} \cdot \mathrm{p}$ model in which every wavefunction is represented by a linear combination of envelope functions, which have approximately the same spatial span of the nanostructure, multiplied by involute wavefunctions that have the same symmetry and span as the atoms in the crystal. In zincblende materials such as GaAs and InAs, the involutes can be labelled according to their point symmetry. The $|S\rangle$ involute has the energy of the $\mathrm{CB}$ bottom, whereas the $|X\rangle,|Y\rangle$ and $|Z\rangle$ involutes 


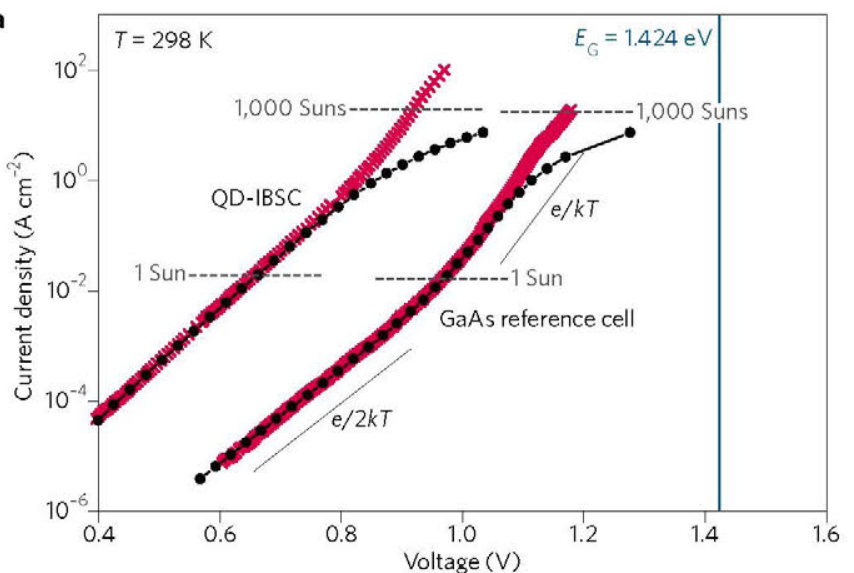

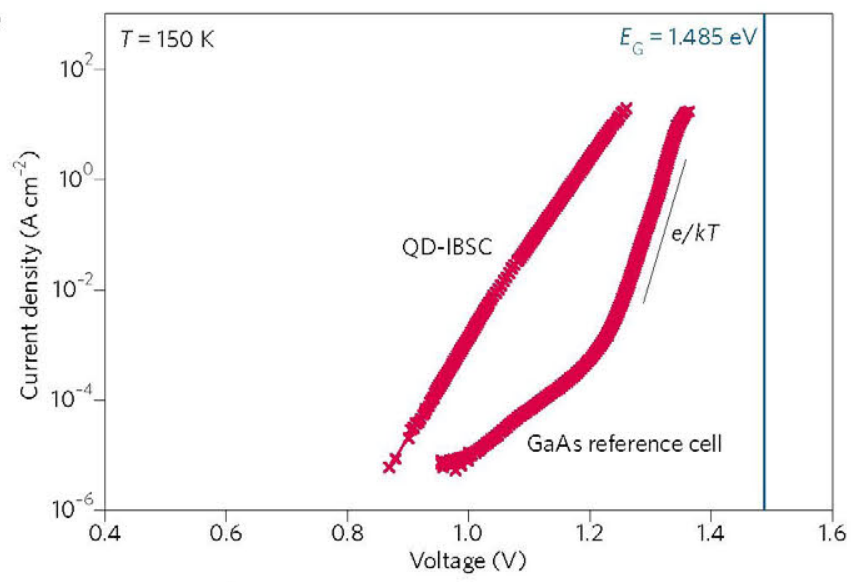

$*-1-V_{\text {oC }}$ under concentration $\rightarrow$ Dark $J-V$
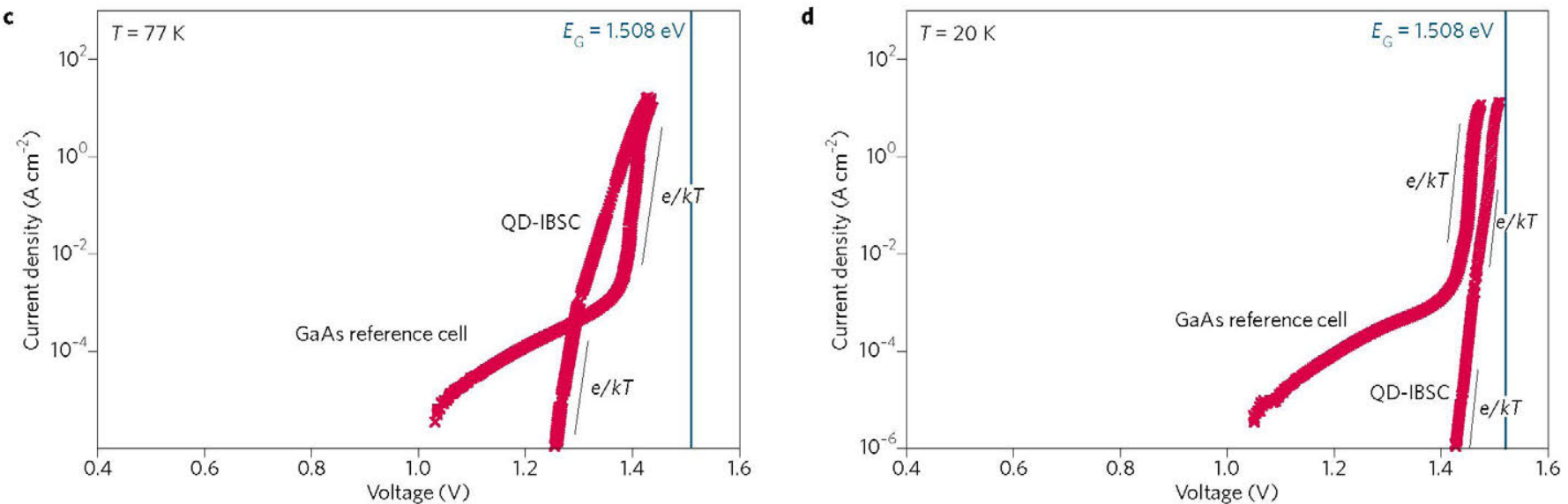

Figure 3 | Open-circuit voltage $\left(V_{O C}\right)$ of a QD IB solar cell as a function of photogenerated current density $\left(I_{L}\right)$ at a range of temperatures. $a$, Dark $I-V$ (black dots) and concentrated-light $I_{L}-V_{O C}$ (red crosses) characteristics of both the QD IB solar cell and the reference cell at room temperature (the difference usually shows resistive effects). The concentration levels are indicated with horizontal dashed lines. b-d, Concentrated-light $I_{L}-V_{0 c}$ measurements at temperatures of $150 \mathrm{~K}(\mathbf{b}), 77 \mathrm{~K}(\mathbf{c})$ and $20 \mathrm{~K}(\mathbf{d})$. The solar cells' fundamental bandgap is represented with a solid, blue line for each temperature, and the e $/ k T$ and $e / 2 k T$ exponential slopes are also indicated, when present. Figure reproduced with permission from ref. 28 , (c) $2012 \mathrm{Elsevier}$.

have energies close to the VB top. In a nanostructured material, the linear combination that corresponds to $\mathrm{CB}$ confined states is almost entirely projected $\left(91 \%\right.$ for the $\mathrm{CB}(1,1,1)$ state $\left.{ }^{25}\right)$ onto the $|S\rangle$ involute, thus confirming the validity of the one-band $\mathrm{k} \cdot \mathrm{p}$ approximation for $\mathrm{CB}$ states. In contrast, the $\mathrm{VB}$ wavefunctions have (in this approximation) zero projection on the $|S\rangle$ involute, and are therefore entirely projected onto the $|X\rangle,|Y\rangle$ and $|Z\rangle$ involutes. Equal-probability surfaces of the envelope functions are shown in Fig. 2c for the $\mathrm{CB}(1,1,1)$ state (the IB) and the $\mathrm{HH}(1,1,1)$ state (the VB state with the highest energy). The green box represents the QD. The dimensions of the QDs used in the representative calculations correspond to QDs with a base length of $16 \mathrm{~nm}$ and a height of $6 \mathrm{~nm}$.

Figure 2e plots the photon absorption coefficient for transitions between the confined VB states and each confined CB state (indicated by the label). This absorption coefficient accurately explains the experimental observations of the sub-bandgap photocurrent ${ }^{25}$. The only transitions to the IB are the ones to the $\mathrm{CB}(1,1,1)$ state. Our present understanding indicates that transitions to other confined states in the $C B$ are found to relax quickly to this $(1,1,1)$ state because they are strongly connected among themselves by thermal infrared photons ${ }^{24}$.

It is important to note that the above results assume the dots to be three-dimensional boxes. More accurate calculations requiring heavier computations of the electronic states in the QDs have also been performed $\mathrm{d}^{20,26}$, which agree reasonably with the simplified calculations summarized in the previous paragraphs.

For an optical transition to occur, the wavefunctions of the initial and final states must have non-null projections on at least one common involute function. If this is not the case, different symmetries of the involute functions will cause the transition matrix element to vanish. Because the $\mathrm{CB}$ confined states project mainly onto the $|S\rangle$ involute, whereas the states in the VB project onto the rest of the involutes, the matrix elements for the interband $\mathrm{VB}-\mathrm{CB}$ transitions (including VB-IB) are small and so the absorption is weak. In contrast, intraband transitions from $\mathrm{CB}$ states to other $\mathrm{CB}$ states nearly always project onto the same involute, resulting in a strong matrix element. The relatively low values for the intraband absorption coefficients in Fig. $2 \mathrm{~d}$ are due to the fact that they refer only to transitions between the IB state, which is half-filled with electrons, and the remaining small number of confined $\mathrm{CB}$ states.

Preservation of voltage. All the QD IB solar cells manufactured so far have exhibited a loss of voltage at room temperature with respect to control cells without QDs. Decreasing the temperature and therefore reducing thermal escape from the $\mathrm{IB}$ to the $\mathrm{CB}$ might not be sufficient to preserve the output voltage if tunnelling still allows electrons to escape from the confined states to the continuum, in particular when QDs are located in a region of electric field such as the space-charge 
a

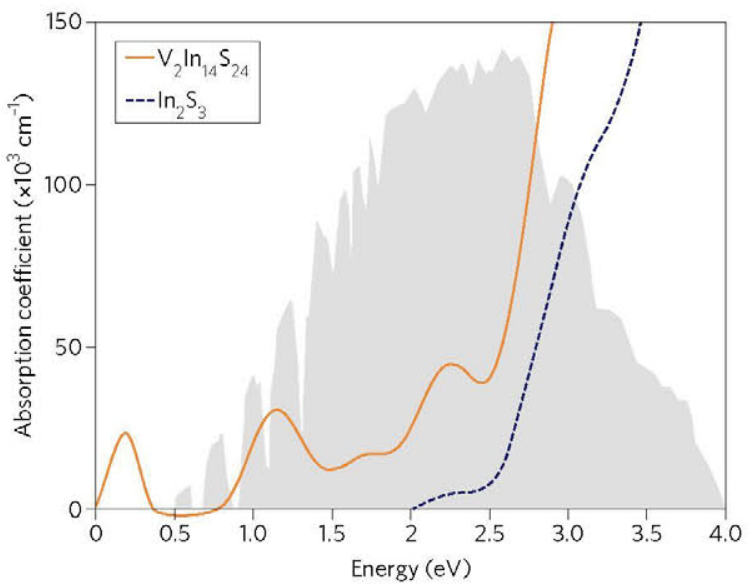

c

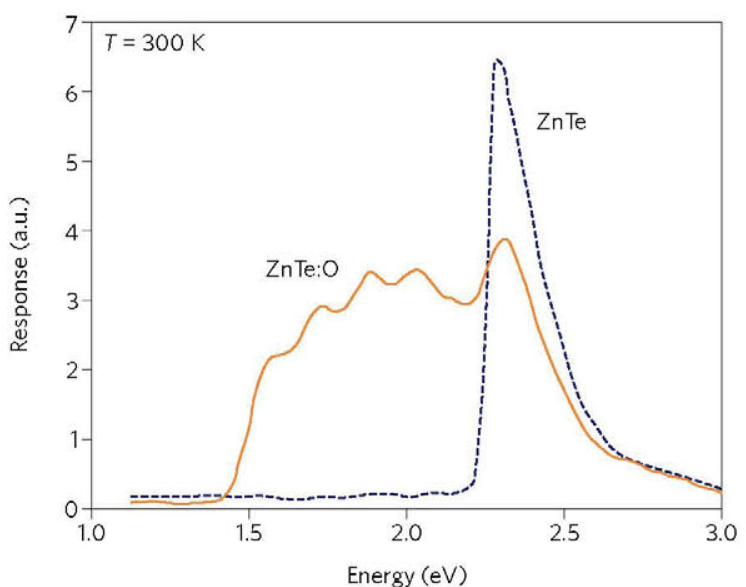

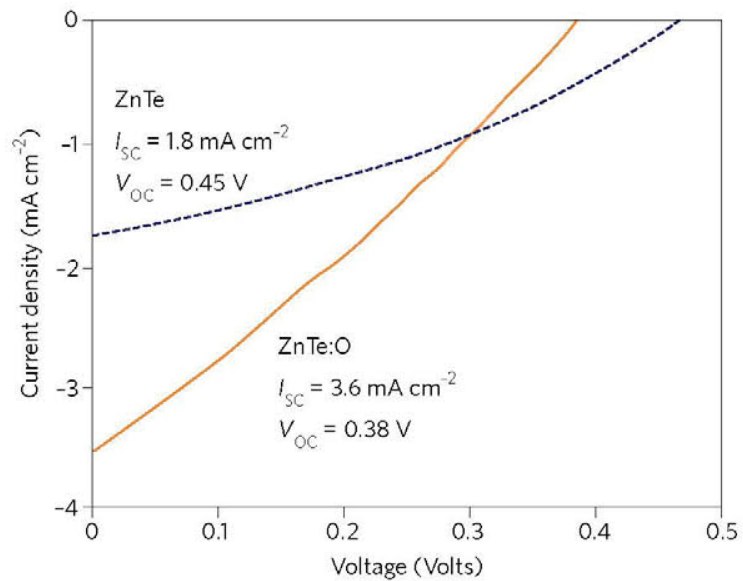

d

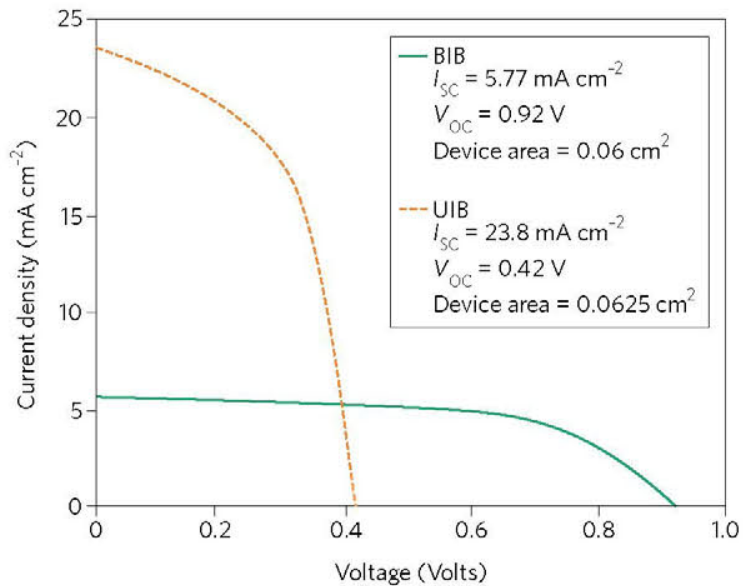

Figure 4 | Results related to the implementation of IB solar cells based on bulk approaches. a, Absorption coefficient of indium sulphide with a vanadium IB material. $\mathbf{b}$, Current-voltage curve of a ZnTe:O solar cell compared with that of a ZnTe solar cell. c, Quantum efficiency of a ZnTe:O solar cell compared with that of a ZnTe solar cell. d, Current-voltage curve of a GaNAs IB solar cell. BIB, blocked intermediate band; UIB, unblocked intermediate band. Figure reproduced with permission from: a, ref. 38, (c) 2008 ACS; b,c, ref. 45, (c) 2009 AIP; d, ref. 47, (c) 2011 APS.

region. However, placing thick spacers (around $80 \mathrm{~nm}-$ much larger than the traditional value of $20 \mathrm{~nm}$ ) between QD layers removes tunnelling ${ }^{27}$ and thus preserves or even increases ${ }^{28}$ the voltage at low temperatures. This can be seen in Fig. 3 for a temperature of $20 \mathrm{~K}$.

At room temperature, the several $\mathrm{CB}$ levels confined within the bandgap ${ }^{23}$ are connected strongly to the IB level by thermal photons ${ }^{24}$, and perhaps also by phonons. Shockley-Read-Hall (SRH) recombination ${ }^{29,30}$ is a mechanism present in most semiconductor devices that restores thermal equilibrium by de-exciting electrons in higher-energy states, for example those in the $\mathrm{CB}$, and dissipating their excess energy by the emission of several phonons. This mechanism is linked to deep impurities, which have energy levels situated in the middle of the bandgap and are active even at rather small concentrations. The SRH mechanism can also operate for localized states in the $\mathrm{CB}$, and therefore for localized states in the IB. The difference, however, is that the associated bandgap for states in the IB is the $E_{\mathrm{H}}$ sub-bandgap of IB solar cells, instead of the bandgap $E_{\mathrm{G}}$ in ordinary cells, for example. Because $E_{\mathrm{H}}<E_{\mathrm{G}}$, the recombination becomes stronger and the open-circuit voltage, which indicates the level of excited electrons that the cell can maintain to equate the recombination with photon pumping, decreases.

Decreasing the temperature reduces the number of thermal photons and phonons, and the IB-CB QFL splits. For a given voltage (determined by the CB-VB split), the appearance of the IB-CB split reduces the IB-VB QFL split and thus weakens SRH recombination from the IB to the VB. The cell operates with the three QFLs, split as required for $\mathrm{IB}$ cell behaviour, and the total voltage of the cell approaches that of the reference cell. The signature for this behaviour is the convergence between IB and test cell curves ${ }^{28}$ (Fig. 3).

\section{Bulk IB materials and solar cells}

Bulk IB materials. Numerous $a b$ initio quantum calculations have been performed in the quest to realize IB materials ${ }^{31-34}$, beginning (to our knowledge) with the work of Wahnon and Tablero ${ }^{31}$. Walukiewicz and co-workers ${ }^{35}$ were the first to synthesize a bulk IB material, $\mathrm{Zn}_{0.88} \mathrm{Mn}_{0.12} \mathrm{Te}_{0.987} \mathrm{O}_{0.013}$. The researchers detected the IB by spectral photoreflectance measurements and ascribed its formation to band anticrossing ${ }^{36}$, which occurs, according to the authors, in heavily mismatched alloys. A few years later, the same authors reported IB behaviour in $\mathrm{GaN}_{x} \mathrm{As}_{1-x-y} \mathrm{P}_{y}$ alloys with $y>0.3$, where the IB was once again detected by photoreflectance ${ }^{37}$. The material was prepared by heavy ion implantation and pulsed laser melting annealing.

$A b$ initio calculations ${ }^{33}$ suggest that $\mathrm{V}_{0.25} \mathrm{In}_{1.75} \mathrm{~S}_{3}$ also has an IB containing the Fermi level. This is important because it means the IB is already partially filled without having to rely on impurity doping, which reduces densities and therefore lowers absorption levels. This material, which researchers have been able to synthesize in powder 
form by chemical solvo-thermal methods, has demonstrated impressively high sub-bandgap absorption ${ }^{38}$ (Fig. 4a).

Silicon heavily doped with titanium has also been prepared by ion implantation and pulsed laser melting annealing. The resulting material is monocrystalline and has a conductivity very close to the non-implanted value. Under measurements at several temperatures, this material has demonstrated electrical behaviour identical to that of an IB-n junction ${ }^{39}$.

Suppressing non-radiative recombination. Although several nonradiative recombination mechanisms may exist, we believe that the dominant process in IB solar cells is that described by Lang and Henry ${ }^{40}$ to explain SRH recombination. The transition of an extended $\mathrm{CB}$ electron to a localized impurity state, according a configuration diagram that takes into account lattice vibrations ${ }^{41}$, causes the impurity level to cross the $\mathrm{CB}$, which makes the transition highly probable. The energy of the electron is therefore transformed into potential energy of the impurity in a single step. This energy is released with the emission of multiple phonons, thereby achieving an energy balance. This can be prevented if the impurity states are extended ${ }^{42}$, as is the case when the density of the impurities is high enough to exceed the Mott transition ${ }^{43}$, which delineates the insulating and conductive behaviour of the IB.

This effect is counterintuitive because it reduces SRH recombination by increasing the density of impurities, which increases SRH recombination. However, experiments have shown that implanting larger doses of titanium in silicon results in lower SRH recombination ${ }^{4}$.

Bulk IB solar cells. The first bulk IB solar cell was made, to our knowledge, by Phillips and co-workers ${ }^{45,46}$ using $\mathrm{ZnTe}$ doped with oxygen impurities to form the IB. The current-voltage curve of this device is shown in Fig. $4 \mathrm{~b}$ together with that of a ZnTe reference cell without oxygen. The cell generates both a higher current and a higher efficiency than the reference cell, although at a lower output voltage. The quantum efficiency (the ratio of electrons out to photons in) of the device is presented in Fig. 4c, demonstrating clear sub-bandgap absorption in the cell with oxygen. Walukiewicz and co-workers analysed two distinct cell structures of a bulk GaNAs IB solar cell ${ }^{47}$ : a 'blocked IB' cell, in which an n-AlGaAs blocking layer is introduced to isolate the IB from the n-emitter; and an 'unblocked IB' reference cell, in which this layer is not introduced. The unblocked IB structure gave a high current but low voltage. The researchers attributed the low voltage to the back cell structure, which short-circuits the IB-CB QFLs and prevents a larger voltage from being achieved. In contrast, they observed a dramatic increase in voltage for the blocked IB cell, although with a considerable loss of photocurrent. The importance of this device has already been stressed ${ }^{48}$.

\section{Summary and challenges}

The addition of InAs QDs to a GaAs solar cell makes the resulting device operate according to the principles of an IB solar cell below room temperature. This generates the sub-bandgap photocurrent and preserves the voltage when the three QFLs split. To observe this behaviour at room temperature, the confined states between the IB and the $\mathrm{CB}$ must be removed and the bandgap enlarged. The QDs must therefore be much smaller than current dimensions - of the order of $5 \mathrm{~nm}$. To this end, researchers have proposed a change in the way QDs are manufacture ${ }^{49}$; from a technique based on strain to one relying on the immiscibility between different crystalline structures (for example, zincblende of CdTe and rock-salt PbTe). QDs of the desired size (down to $2-3 \mathrm{~nm}$ ) have been produced through this technique ${ }^{50}$ and can be implemented with several material options that give a bandgap distribution close to the optimum value of $1.95 \mathrm{eV}$, with good lattice matching. Nevertheless, some carefully manufactured InAs/GaAs cells have already presented minor voltage reductions ${ }^{18,19}$ and are therefore probably already operating as IB cells at room temperature.

The issue of low VB-IB photon absorption can be solved primarily by increasing the density of the QDs. This has already been shown to be true for InAs/GaAs structures ${ }^{20}$. Further experimental work is required with immiscible materials, although it is encouraging to note that high densities do seem to be feasible in this technique ${ }^{50}$.

Increasing the density of InAs QDs in GaAs would lead to a reasonable absorptance. For instance, using QD spacers with a thickness of $20 \mathrm{~nm}$ (as in most of the experimental work referenced here) instead of $80 \mathrm{~nm}$ (as in the tunnel-free cell ${ }^{27,28}$ ), together with a larger QD surface density ${ }^{21}$ (surface coverage of $50 \%$ instead of $10 \%$ in the cell analysed), would cause $\mathrm{e}^{25}$ the sub-bandgap current to increase to around $4.4 \mathrm{~mA} \mathrm{~cm}^{-2}$. However, the absorptance value that corresponds to this sub-bandgap current is not yet at the desirable value of unity. This ideal value could be approached through the use of gratings to diffract the photons and make them propagate in directions laterally along the solar cell. In the case of smaller QDs in immiscible materials, the interband matrix elements discussed above may increase due to the appearance of further terms that are negligible in the large QDs used so $\mathrm{far}^{51}$.

Tunnelling between QDs has prevented QD-based IB solar cells from reaching high voltages, even at low operating temperatures. It is only necessary to avoid tunnelling between QDs when they are located in regions of electric field, such as at the edges of the QD region. The QD density in the useful centre region could therefore be increased by employing thick spacers in the edge regions while using thinner ones in the neutral centre zone. Doping has been applied successfully as an alternative to this technique ${ }^{11}$.

Researchers have synthesized several bulk IB materials so far. The most promising of these is $\mathrm{V}_{0.25} \operatorname{In}_{1.75} \mathrm{~S}_{3}$, which has strong sub-bandgap absorption and an inherent partially filled IB. Unfortunately, solar cells have not yet been made from this alloy. However, $\operatorname{In}_{2} \mathrm{~S}_{3}$ is already being used as a buffer layer ${ }^{52}$ in some thin-film solar cells, so its adaptation to the manufacture of an IB solar cell should be possible in principle.

Bulk IB solar cells have so far exhibited efficiencies far below their potential, although this is characteristic of test cells without an IB due to their non-optimum bandgap. Mastering the technology of these new materials is essential if IB solar cell research based on them is to succeed, and this is not a trivial task. It must be remembered that it has taken around 60 years for thin-film solar cells to reach their present state of maturity.

In summary, the shortcomings of InAs/GaAs QD solar cells are quite well understood, and we believe this knowledge will guide research towards more promising QD structures. Our vision is that IB solar cells will be commercialized, thereby offering a simpler but equivalent device to the sextuple junction used in concentrator solar cell systems, with efficiencies reaching $50 \%$. We also anticipate that bulk IB solar cells might exceed the efficiencies of current thinfilm solar cells. The concept of an IB solar cell can be extended in several ways to the fertile field of organic semiconductors, again with the promise of increased efficiency. The challenges ahead are not negligible, but the prize is worth the effort.

\section{References}

1. Luque, A. \& Martí, A. A metallic intermediate band high efficiency solar cell. Prog. Photovolt. Res. Appl. 9, 73-86 (2001).

2. Luque, A. \& Martí, A. Increasing the efficiency of ideal solar cells by photon induced transitions at intermediate levels. Phys. Rev. Lett. 78, 5014-5017 (1997).

3. Shockley, W. \& Queisser, H. J. Detailed balance limit of efficiency of $p-n$ junction solar cells. J. Appl. Phys. 32, 510-519 (1961). 
4. Green, A. M. Multiple band and impurity photovoltaic solar cells: General theory and comparison to tandem cells. Prog. Photovolt. Res. Appl.9, 137-144 (2001).

5. Wolf, M. Limitations and possibilities for improvements of photovoltaic solar energy converters. Part I: Considerations for earth's surface operation. Proc. IRE 48, 1246-1263 (1960).

6. Ekins-Daukes, N. J., Honsberg, C. B. \& Yamaguchi, M. Signature of intermediate band materials from luminescence measurements in Proc. 31st IEEE Photovoltaic Specialists Conf. 49-54 (IEEE, 2005).

7. Levy, M. Y. \& Honsberg, C. Solar cell with an intermediate band of finite width. Phys. Rev. B 78, 165122 (2008).

8. Strandberg, R. \& Reenaas, T. W. Photofilling of intermediate bands. J. Appl. Phys. 105, 124512 (2009).

9. Martí, A., Cuadra, L. \& Luque, A. Quantum dot intermediate band solar cell in Proc. 28th IEEE Photovoltaics Specialists Conf. 904-943 (IEEE, 2000).

10. Luque, A. et al. General equivalent circuit for intermediate band devices: Potentials, currents and electroluminescence. J. Appl. Phys. 96, 903-909 (2004).

11. Marti, A. et al. Elements of the design and analysis band solar of quantum-dot intermediate cells. Thin Solid Films 516, 6716-6722 (2008).

12. Hubbard, S. M. et al. Effect of strain compensation on quantum dot enhanced GaAs solar cells. Appl. Phys. Lett. 92, 123512 (2008).

13. Oshima, R., Takata, A. \& Okada, Y. Strain-compensated InAs/GaNAs quantum dots for use in high-efficiency solar cells. Appl. Phys. Lett. 93, 083111 (2008).

14. Kechiantz, A. M., Sun, K. W., Kechiyants, H. M. \& Kocharyan, L. M. Selfordered $\mathrm{Ge} / \mathrm{Si}$ quantum dot intermediate band photovoltaic solar cells. Int. Sci. J. Ait. Energ. Ecol. 12, 85-87 (2005).

15. Laghumavarapu, R. B. et al. Improved device performance of InAs/GaAs quantum dot solar cells with $\mathrm{GaP}$ strain compensation layers. Appl. Phys. Lett. 91, 243115 (2007).

16. Zhou, D., Sharma, G., Thomassen, S. F., Reenaas, T. W. \& Fimland, B. O. Optimization towards high density quantum dots for intermediate band solar cells grown by molecular beam epitaxy. Appl. Phys. Lett. 96, 061913 (2010).

17. Blokhin, S. A. et al. AlGaAs/GaAs Photovoltaic cells with an array of InGaAs QDs. Semiconductors 43, 514-518 (2009).

18. Bailey, C. G., Forbes, D. V., Raffaelle, R. P. \& Hubbard, S. M. Near 1 V open circuit voltage InAs/GaAs quantum dot solar cells. Appl. Phys. Lett. 98, 163105 (2011)

19. Guimard, D. et al. Fabrication of InAs/GaAs quantum dot solar cells with enhanced photocurrent and without degradation of open circuit voltage. Appl. Phys. Lett. 96, 203507 (2010).

20. Popescu, V., Bester, G., Hanna, M. C., Norman, A. G. \& Zunger, A. Theoretical and experimental examination of the intermediate-band concept for strainbalanced (In, Ga)As/Ga(As, P) quantum dot solar cells. Phys. Rev. B 78, 205321 (2008).

21. Akahane, K. et al. Highly packed InGaAs quantum dots on GaAs(311)B. Appl. Phys. Lett. 73, 3411-3413 (1998).

22. Datta, S. Quantum Phenomena Ch. 6 (Addison Wesley, 1989).

23. Luque, A., Marti, A., Antolin, E. \& Garcia-Linares, P. Intraband absorption for normal illumination in quantum dot intermediate band solar cells. Sol. Energ. Mater. Sol. C. 94, 2032-2035 (2010).

24. Luque, A. et al. Radiative thermal escape in intermediate band solar cells. AIP Advances 1, 022125 (2011).

25. Luque, A. et al. New Hamiltonian for a better understanding of the quantum dot intermediate band solar cells. Sol. Energ. Mater. Sol. C. 95, 2095-2101 (2011).

26. Tomic, S., Jones, T. S. \& Harrison, N. M. Absorption characteristics of a quantum dot array induced intermediate band: Implications for solar cell design. Appl. Phys. Lett. 93, 263105 (2008).

27. Antolín, E. et al. Reducing carrier escape in the InAs/GaAs quantum dot intermediate band solar cell. J. Appl. Phys. 108, 064513 (2010).

28. Linares, P. G. et al. Voltage recovery in intermediate band solar cells. Sol. Energ. Mater. Sol. C. 98, 240-244 (2012).
29. Shockley, W. \& Read, W. T. Statistics of the recombination of holes and electrons. Phys. Rev. 87, 835-842 (1952).

30. Hall, R. N. Electron-hole recombination in germanium. Phys. Rev. 87, 387 (1952)

31. Wahnón, P. \& Tablero, C. Ab initio electronic structure calculations for metallic intermediate band formation in photovoltaic materials. Phys. Rev. B 65, 155115 (2002).

32. Palacios, P., Wahnón, P., Pizzinato, S. \& Conesa, J. C. Energetics of formation of $\mathrm{TiGa}_{3} \mathrm{As}_{4}$ and $\mathrm{TiGa}_{3} \mathrm{P}_{4}$ intermediate band materials. J. Chem. Phys. 124, 14711-14715 (2006).

33. Palacios, P., Aguilera, I., Sanchez, K., Conesa, J. C. \& Wahnon, P. Transitionmetal-substituted indium thiospinels as novel intermediate-band materials: Prediction and understanding of their electronic properties. Phys. Rev. Lett. 101, 046403 (2008).

34. Sanchez, K., Aguilera, I., Palacios, P. \& Wahnon, P. Assessment through firstprinciples calculations of an intermediate-band photovoltaic material based on Ti-implanted silicon: Interstitial versus substitutional origin. Phys. Rev. B 79, 165203 (2009).

35. Yu, K. M. et al. Diluted II-VI oxide semiconductors with multiple band gaps. Phys. Rev. Lett. 91, 246403-246404 (2003).

36. Walukiewicz, W. et al. Interaction of localized electronic states with the conduction band: Band anticrossing in II-VI semiconductor ternaries. Phys. Rev. Lett. 85, 1552-1555 (2000).

37. Yu, K. M. et al. Multiband GaNAsP quaternary alloys. Appl. Phys. Lett. 88, 092110-092113 (2006).

38. Lucena, R., Aguilera, I., Palacios, P., Wahnon, P. \& Conesa, J. C. Synthesis and spectral properties of nanocrystalline $\mathrm{v}$-Substituted $\operatorname{In}_{2} \mathrm{~S}_{3}$ : A novel material for more efficient use of solar radiation. Chem. Mater. 20, 5125-5127 (2008).

39. Gonzalez-Diaz, G. et al. Intermediate band mobility in heavily titanium-doped silicon layers. Sol. Energ. Mater. Sol. C. 93, 1668-1673 (2009).

40. Lang, D. V. \& Henry, C. H. Nonradiative recombination at deep levels in GaAs and GaP by lattice-relaxation multiphonon emission. Phys. Rev. Lett. 35, $1525-1528$ (1975).

41. Markvart, T. Multiphonon recombination in Recombination in semiconductors (ed. Landsberg, P. T.) 450 (Cambridge University Press, 1991).

42. Luque, A., Martí, A., Antolín, E. \& Tablero, C. Intermediate bands versus levels in non-radiative recombination. Physica B 382, 320-327 (2006).

43. Mott, N. F. Metal-insulator transition. Rev. Mod. Phys. 40, 677-683 (1968).

44. Antolin, E. et al. Lifetime recovery in ultrahighly titanium-doped silicon for the implementation of an intermediate band material. Appl. Phys. Lett. 94, 042115 (2009).

45. Wang, W., Lin, A. S. \& Phillips, J. D. Intermediate-band photovoltaic solar cell based on ZnTe:O. Appl. Phys. Lett. 95, 011103 (2009).

46. Wang, W., Lin, A. S., Phillips, J. D. \& Metzger, W. K. Generation and recombination rates at $\mathrm{ZnTe}: \mathrm{O}$ intermediate band states. Appl. Phys. Lett. 95, 261107 (2009).

47. Lopez, N., Reichertz, L. A., Yu, K. M., Campman, K. \& Walukiewic, W. Engineering the electronic band structure for multiband solar cells. Phys. Rev. Lett. 106, 028701 (2011).

48. Luque, A. \& Marti, A. Photovoltaics: Towards the intermediate band. Nature Photon. 5, 137-138 (2011).

49. Antolín, E., Martí, A. \& Luque, A. The lead salt quantum dot intermediate band solar cell in Proc. 37th Photovoltaic Specialists Conf. (IEEE, 2011).

50. Heiss, W. et al. Quantum dot with coherent interfaces between rocksalt-PbTe and zincblende-CdTe. J. Appl. Phys. 101, 081723 (2007).

51. Harrison, P. Quantum Wells, Wires and Dots 333 (Wiley, 2005).

52. Barreau, N. et al. Study of a new indium sulphide derivative for buffer layer application in Proc. 29th IEEE Photovoltaic Specialists Conf. 628-631 (IEEE, 2002).

53. Martí, A. et al. Emitter degradation in quantum dot intermediate band solar cells. Appl. Phys. Lett. 90, 233510 (2007). 